\title{
BasicBayes: A tutor system for simple Bayesian inference
}

\author{
PETER SEDLMEIER \\ Universität Paderborn, Paderborn, Germany
}

\begin{abstract}
To date, attempts to teach Bayesian inference to nonexperts have not met with much success. BasicBayes, the computerized tutor presented here, is an attempt to change this state of affairs. BasicBayes is based on a novel theoretical framework about Bayesian reasoning recently introduced by Gigerenzer and Hoffrage (1995). This framework focuses on the connection between "cognitive algorithms" and "information formats." BasicBayes teaches people how to translate Bayesian text problems into frequency formats, which have been shown to entail computationally simpler cognitive algorithms than those entailed by probability formats. The components and mode of functioning of BasicBayes are described in detail. Empirical evidence demonstrates the effectiveness of BasicBayes in teaching people simple Bayesian inference. Because of its flexible system architecture, BasicBayes can also be used as a research tool.
\end{abstract}

Estimating the probability of a hypothesis in the face of new data is a task with which we all are confronted every day. A general framework for making such judgments is provided by Bayesian inference. For more than two decades, a considerable amount of research has been devoted to finding out whether people apply Bayesian inference in tasks in which it is applicable. The results have turned out to be overwhelmingly negative. In particular, it has been found that people apparently neglect the prior probability of hypotheses - that is, their base rates. The following conclusion from Bar-Hillel (1980) represents those of most researchers in this area of research well: "The genuineness, the robustness, and the generality of the baserate fallacy are matters of established fact" (p. 215).

Here is an abbreviated example of the kind of problem that is typically used in research on Bayesian inferencethe "mammography problem" (adapted from Casscells, Schoenberger, \& Grayboys, 1978, and Eddy, 1982):

The prevalence of breast cancer is $1 \%$ for women over age 40. A widely used test, mammography, gives a positive result in $10 \%$ of women without breast cancer, and in $80 \%$ of women with breast cancer. What is the probability that a woman in this age class who tests positive actually has breast cancer?

The vast majority of people presented with problems like this one gives incorrect solutions. In a study by Eddy (1982), for instance, 95 out of 100 physicians given num-

This research was supported by a Feodor Lynen Stipend of the Humboldt Foundation as well as a Habilitationsstipendium of the Deutsche Forschungsgemeinschaft. I thank Valerie Chase, Ralph Hertwig, Detlef Köhlers, Jürgen Locher, and Manfred Wettler for many constructive comments; Jim Magnusson and Tom McDougal for helping to get the project started; and Gerd Gigerenzer for his continuous support. Correspondence should be addressed to P. Sedlmeier, Universität Paderborn, Fachbereich 2-Psychologie, 33095 Paderborn, Germany (e-mail: sedl@) psycho.uni-paderborn.de). erical information nearly identical to that above estimated the probability $p$ (cancer $\mid$ positive) to be $70 \%-80 \%$.

What would have been the Bayesian solution? The three crucial pieces of information stated in the text (percentages transformed into probabilities) are:

$$
\begin{aligned}
& \text { base rate: } p(\text { cancer })=.01 \\
& \text { hit rate: } p(\text { positive } \mid \text { cancer })=.80 \\
& \text { false alarm rate: } p(\text { positive } \mid \text { no cancer })=.10
\end{aligned}
$$

The mammography problem can be solved by applying Bayesian inference in its most elementary form with two exhaustive and exclusive (point) hypotheses, "cancer" and "no cancer." (Only this simple form of Bayesian inference is dealt with here.) The task is to estimate the posterior probability $p$ (cancer|positive). The version of Bayes's theorem needed to compute this probability from the given probabilities is shown at the bottom of the next page. Thus, the Bayesian posterior probability is an order of magnitude smaller than the modal estimate typically found in experimental studies of the mammography problem.

This paper first gives a short overview of previous attempts to teach Bayesian reasoning. The theoretical basis for BasicBayes, a novel theoretical framework for statistical reasoning introduced by Gigerenzer and Hoffrage (1995), is then presented. Finally, the components of BasicBayes are described in detail.

\section{TEACHING BAYESIAN INFERENCE}

Shaughnessy (1992, p. 469) wonders why cognitive psychologists lament the depth and tenacity of people's misconceptions of probability, and at the same time make little attempt to collaborate with mathematics educators in designing effective ways to teach probabilistic reasoning. Indeed, there have been only a few attempts on the part of 
cognitive psychologists to teach Bayesian reasoning to nonexperts, most of them based on corrective feedback (Lindeman, van den Brink, \& Hoogstraten, 1988; Peterson, DuCharme, \& Edwards, 1968; Schaefer, 1976). No substantial training effect was found in any of these studies.

In a different kind of training study, Fong, Lurigio, and Stalans (1990) taught participants to be sensitive to the effect of sample size on the accuracy of estimates. They expected that training would also have beneficial effects on Bayesian reasoning by directing participants' attention to base rates. Their study assumes that rule trainingthat is, training in formal aspects of statistical lawsenhances statistical reasoning (Nisbett, 1993). Its effectiveness was limited, however: Only when the base rate of the outcome to be predicted was very high did the training enhance the use of base rates. The tutoring system presented in this paper, BasicBayes, relies on a different theoretical approach. It assumes that training in the use of external representational formats, rather than rule training, improves Bayesian reasoning.

\section{Representational Formats in Statistical Reasoning}

Recall that the information in the mammography problem was represented in terms of probabilities expressed as percentages. According to Gigerenzer and Hoffrage (1995), information format (e.g., probability vs. frequency) is crucial to how people solve the problem. In order to understand their argument, let us assume that humans have evolved capacities or algorithms for statistical reasoning. Let us further assume that the design of the mind and its environment have evolved in tandem. What would be the input format to which evolved statistical algorithms are tuned? It would almost certainly not be probabilities or percentages, since the concept of probability entered human thinking only several hundred years ago - far too recently to have had a remarkable influence on the evolution of the human mind.

Cosmides and Tooby (1996) and Gigerenzer (1994) argue that the mind's algorithms for statistical reasoning are tuned to frequencies of events rather than probabilities or percentages. This argument explains why researchers in most studies have come to negative conclusions about people's ability to reason statistically: Most of them have employed text problems that state the numerical information in terms of probabilities or percentages. If this explanation is correct, then difficulties in solving statistical reasoning problems should largely disappear if the information is presented in the form of absolute frequencies instead of probabilities or percentages. Indeed, studies on the "conjunction fallacy" (Fiedler, 1988; Hertwig, 1995), "overconfidence bias" (Gigerenzer, Hoffrage, \&
Kleinbölting, 1991), and "base rate neglect" (ChristensenSzalanski \& Beach, 1982; Gigerenzer, Hell, \& Blank, 1988 ) support the idea that people's statistical reasoning improves when information is presented in terms of frequencies.

How would the mammography problem look in a frequency format? We must first specify a reference class (e.g., 100 women) and then express all percentages as absolute frequencies. For example:

Imagine 100 women. Assume that 1 woman has cancer and a positive mammography. Also, assume that there are 10 more women with positive mammographies but no cancer. Thus about 11 women test positive. How many women who test positive have cancer?

Gigerenzer and Hoffrage (1995) translated Bayesian problems into frequency formats similar to the one above and found $46 \%$ spontaneous Bayesian solutions, as compared with $16 \%$ with the same problems stated in terms of percentages. They also analyzed the computations necessary to solve the problem in each format and found that frequency formats entailed computationally simpler Bayesian algorithms than did probability formats. In the transformed mammography problem, for instance, one can immediately "see" the answer 1 out of 11 women by dividing the number of positive tests \& cancer $(\mathrm{P} \& \mathrm{C})$ by the number of positive tests $(\mathrm{P})$. This relative frequency then gives the Bayesian posterior probability: $p$ (cancer $\mid$ positive $)=(\mathrm{P} \& \mathrm{C}) / \mathrm{P}$.

If humans possess cognitive algorithms for Bayesian inference that work on frequency formats, the most effective training would not be to show them Bayes's formula but to teach them how to translate information represented in a probability format into a frequency format. This is precisely what BasicBayes is designed to do.

\section{THE TUTOR}

BasicBayes was adapted from a subject-tested tutor developed by Sedlmeier and Gigerenzer (1995). BasicBayes is an unintelligent tutor: It does not contain a student module that allows for assessment of the student's current level of knowledge or for custom tailoring of the instructional strategy. Instead, it is more in line with approaches to computerized tutoring that emphasize apprenticeship (e.g., Newman, 1989) and rich problem-solving interfaces (e.g., Nathan, Johl, Kintsch, \& Lewis, 1989). More than 10 years of research on "cognitive tutors" has led J. R. Anderson and colleagues to conclude that as the operational definition of the learning task, the problem-solving interface is of primary importance and should even be con-

$$
\begin{aligned}
p(\text { cancer } \mid \text { positive }) & =\frac{p(\text { cancer }) * p(\text { positive } \mid \text { cancer })}{p(\text { cancer }) * p(\text { positive } \mid \text { cancer })+p(\text { no cancer }) * p(\text { positive } \mid \text { no cancer })} \\
& =.01 \times .80 /(.01 \times .80+.99 \times .10) \\
& =.008 / .107 \\
& =.075 .
\end{aligned}
$$


structed first (Anderson, Corbett, Koedinger, \& Pelletier, 1995). Consistent with the theoretical analysis of information formats above, the interface is the most important aspect of BasicBayes.

In their test of BasicBayes's predecessor, Sedlmeier and Gigerenzer (1995) identified two frequency formats that were particularly effective in teaching Bayesian reasoning: the frequency grid and the frequency tree. In the frequency grid, frequencies are displayed pictorially by the number of marked squares in a grid; in the frequency tree, frequencies are displayed by numbers in a tree diagram. On the basis of informal trial sessions, in which just as many participants preferred to work with the frequency tree as with the frequency grid, it was decided that both kinds of frequency format would be employed in BasicBayes. The program was developed on a Macintosh Performa and written in Macintosh CommonLisp (Apple Computer, 1992).

\section{Interface}

In both the frequency grid and the frequency tree training, the screen is always divided into three windows. Figure 1 shows these three windows for the mammography problem during frequency grid training (different screen colors are represented here as different shadings). The problem is shown in the text window (bottom left). The representation window (top) displays a frequency grid representing 1,000 women who have undergone mammography. The tutor window (bottom right) gives information, explanations, and instructions. The user interacts with the program via either the tutor window or the representation window and is allowed to manipulate single squares with the mouse. In the mammography problem, for instance, a woman (represented as a square) can be marked as having breast cancer and/or a positive test result. Depending on current requirements, the tutor window provides several choices that can be selected by mouse, and it permits the user to insert text.

Figure 2 shows the screen during frequency tree training. In this case, the text window appears at the upper right. The user has just been asked to enter two numbers in the tutor window, which yield the desired posterior probability. The left window shows the frequency tree itself with numerical values already filled in. The user can change any value in a node by clicking that node with the mouse and typing.

\section{Knowledge Representation}

BasicBayes represents knowledge about each problem in frame-like structures (see, e.g., Minsky, 1975; Sedlmeier \& Robles de Acuña-Ponseti, 1992) using CLOS (see Keene, 1989). Figure 3 shows the problem representation frame, which consists of 10 slots. One slot is devoted to the text, the question, and the title of the problem, respectively. Several other slots contain the segments of text needed if BasicBayes is called upon to explain the problem to the user. In particular, BasicBayes provides a term for the individuals or objects involved in the problem (e.g., Women), a hypothesis label (e.g., Breast Cancer), and an explanation of true positives (e.g., Positive Test \& Breast

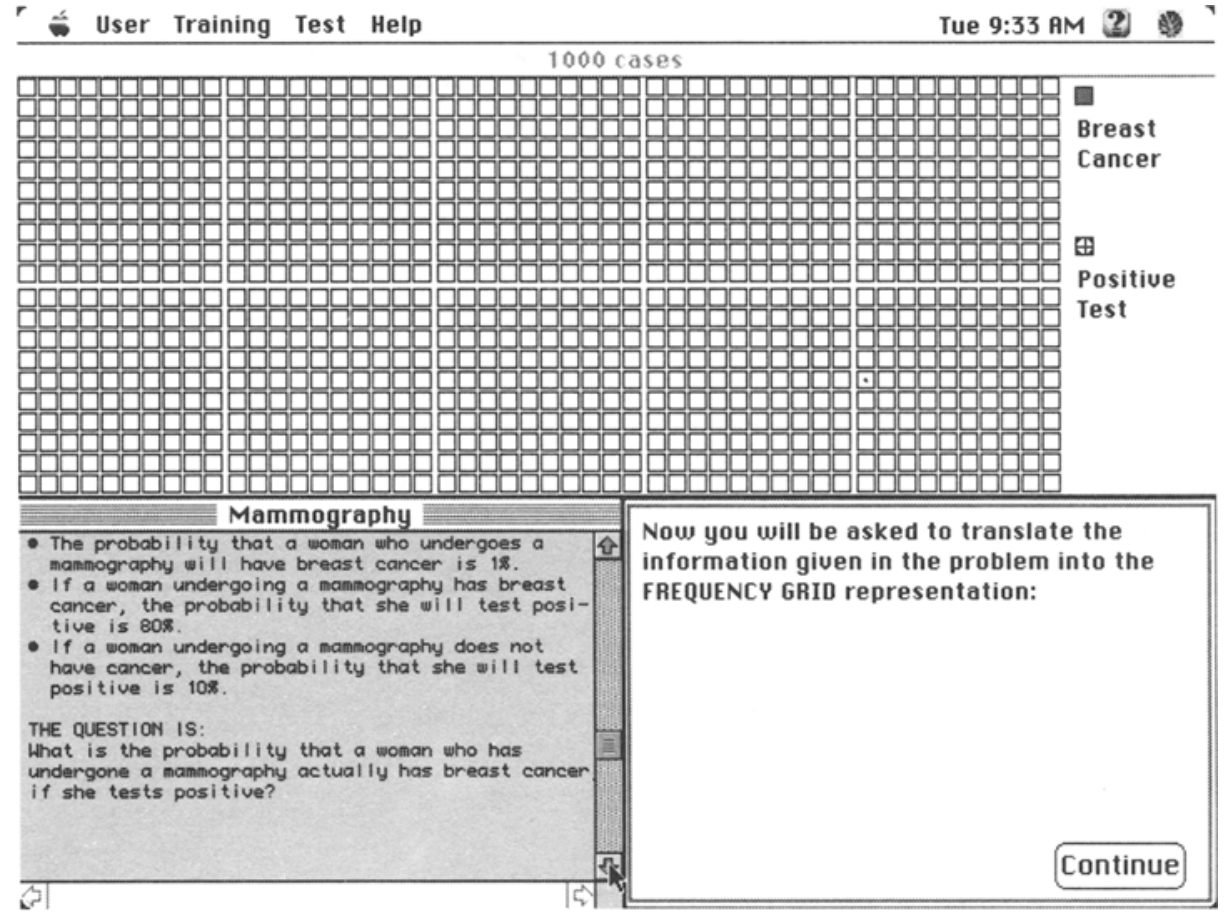

Figure 1. A typical screen during BasicBayes training. The text window (bottom left) shows part of the mammography problem, the representation window (top) displays a $50 \times 20$ frequency grid, and the tutor window (bottom right) gives a preview of what will be the next step in the training. 


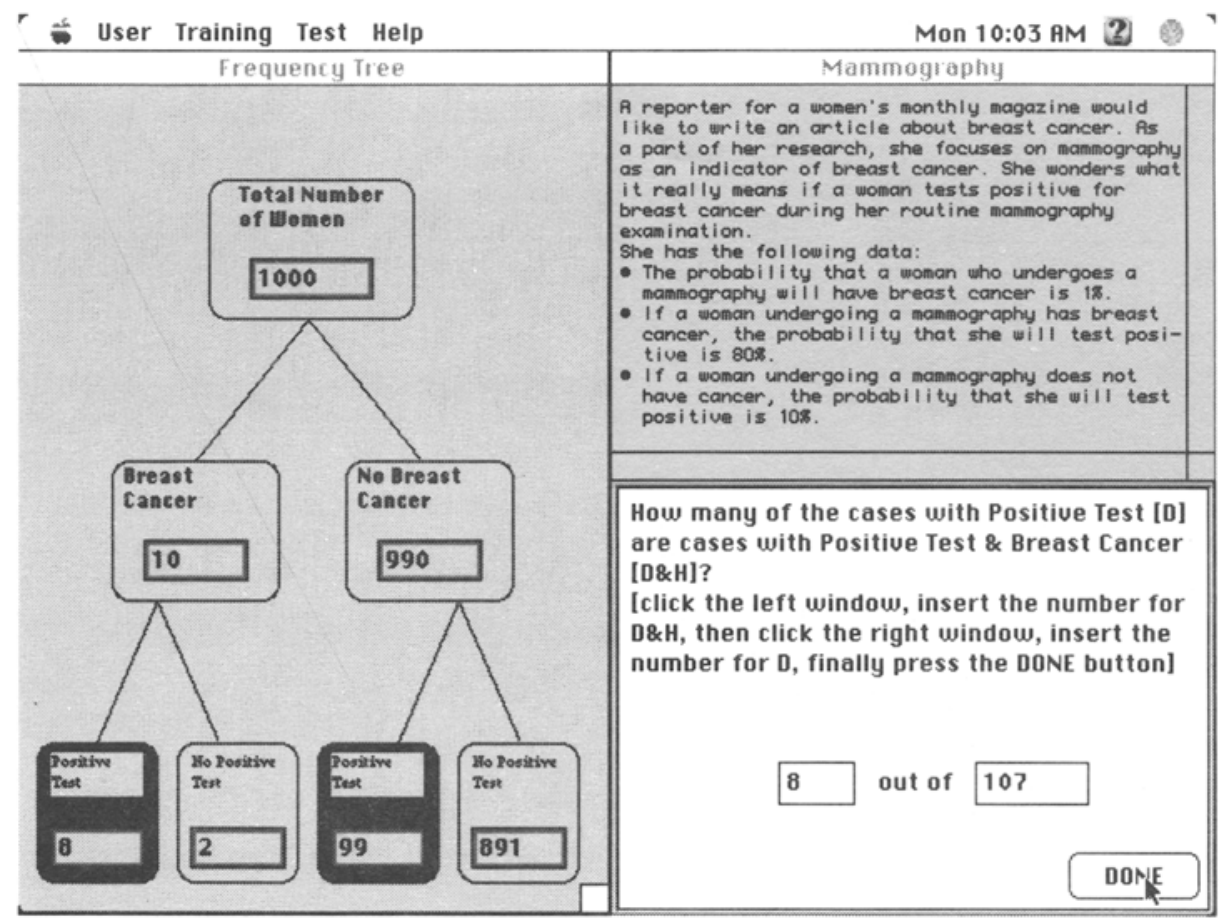

Figure 2. Screen during frequency tree training, just before solution of the mammography problem.

Cancer) and false positives (e.g., Positive Test \& No Breast Cancer) in the problem at hand.

Finally, some slots of the problem representation contain the numerical information given in the problem, which is always expressed in terms of probabilities. If BasicBayes has to deal with frequencies, it multiplies the probabilities by the size of the reference class given by the user. Consider an example involving the frequency tree representation of the mammography problem: If the system has to fill in (or check) a number in the node for the hypothesis (Breast Cancer), it multiplies the given probability $(.01)$ by the size of the relevant reference class (e.g., 1,000$)$ and uses the resulting value $(20)$ for evaluation. If the result is not an integer (as with a small reference class it frequently is not), it rounds both to the next higher and the next smaller integer. The integer closer to the (real) number resulting from multiplication is used for demonstration and explanation, and both are used in the evaluation of user input.

\section{Modes of Teaching}

BasicBayes has two modes of teaching, one fixed and one flexible. In the fixed mode, the program works out detailed solutions to two problems, the sepsis problem and the mammography problem. In the flexible mode, the program allows the user to choose problems that she then has to work out herself. In this mode, the user can fully manipulate the frequency formats shown on the screen. Both modes work with the frequency grid and the frequency tree.
Fixed mode. In the fixed mode, training advances in steps. At each step, BasicBayes explains what it will do next and why. First, it demonstrates how the information in the problem text can be translated into a frequency format and then how one can solve for the desired posterior probability by using this format. Let us demonstrate the fixed mode with the frequency grid version.

The fixed mode begins by explaining and working out a detailed solution to the sepsis problem (not shown here). Figure 4 displays an early point in the solution to the second problem that it works out for the user, the mammography problem. The frequency grid has just been introduced, and BasicBayes begins analyzing the information given in the problem text. It continues by entering the cases referring to the hypothesis (the one woman with breast cancer) into the $10 \times 10$ grid. Because that woman has been correctly diagnosed as having breast cancer ( $80 \%$ of 1 is rounded to 1 ), it then marks that entry with a circled cross. Once the false positives have also been entered, BasicBayes arrives at a first solution (Figure 5).

Since the values in Figure 5 are rounded, the solution is not very accurate. (Recall that plugging the probabilities into Bayes's formula yields a solution of .075.) Therefore, BasicBayes proceeds to illustrate how the size of the reference class can influence the accuracy of the solution. In the mammography problem, the exact numbers of true positives and false positives for the mammography test are .8 ( $80 \%$ of 1 woman) and $9.9(10 \%$ of 99 women). Since the grid accepts only whole cases, the resulting ratio of true positives divided by all positives in a $10 \times 10$ grid 


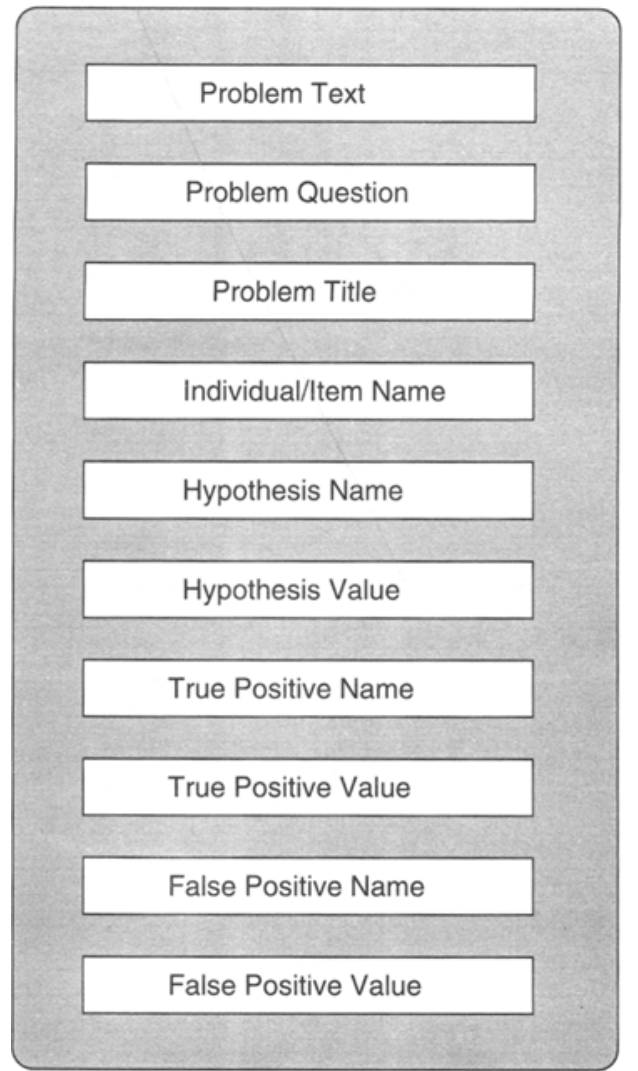

Figure 3. A frame containing BasicBayes's "knowledge" about each problem. See text for further explanation.

is $1 / 11(.09)$. The exact value can be attained by using a $50 \times 20$ grid. Toward the end of the fixed mode training, BasicBayes juxtaposes the different estimates calculated from the two grids in order to demonstrate that using a larger reference class results in a more accurate estimate of the posterior probability (Figure 6).

The frequency tree version follows the same line of reasoning as does the frequency grid version, and the two versions are worded as similarly as possible.

Flexible mode. In the flexible mode, BasicBayes asks the user to perform actions (e.g., shading the grid or entering numbers), gives hints, and delivers immediate feedback to incorrect input. Immediate feedback has proved to be very useful, although not always optimal, in tutor systems (Anderson, Boyle, Corbett, \& Lewis, 1990). Like the fixed mode, the flexible mode is available in both versions of the frequency format. Let us first illustrate this mode in the frequency tree representation mode of the mammography problem (Figure 2).

The user first has to choose the size of the reference class. BasicBayes recommends 100 or 1,000 , but the user can choose any size. A reference class of 1,000 has already been chosen in Figure 2 (top node). The user is instructed how to enter numbers into the nodes of the tree and then to do so. BasicBayes is able to perform several kinds of corrective interventions in response to the user's entries. The user always has a choice between trying again or let- ting BasicBayes perform the corrections. If, after several corrective interventions, the user is still unable to fill in the numbers correctly and does not want to try again, $\mathrm{Ba}-$ sicBayes inserts the correct numbers into all nodes and asks the user to key in the numbers needed to calculate the ratio (H\&D)/D (see Figure 2). BasicBayes accepts only correct solutions and urges users to try again until they succeed.

As in the fixed mode, the frequency grid version is constructed to be as similar as possible to the frequency tree version. Unlike the frequency tree version, however, where the size of the reference class can be chosen without restriction, the frequency grid offers only two sizes, 100 and 1,000. BasicBayes provides two ways to insert (or delete) information in the grid: clicking squares with the mouse (and pressing another key), or keying in the numbers and letting the program fill in the shadings and circled crosses where appropriate. BasicBayes verifies that all the necessary information has been inserted and then checks whether the numbers of shaded squares, shaded and crossed squares, and squares with just a cross coincide with the information given in the problem. If there is no match, it shows the correct numbers on request. Figure 7 illustrates that the numerical entries can be made in any order as long as the number of cases falling into each class $(H, D \& H, D \&$ not $H)$ is entered correctly.

Finally, as in the frequency tree version, the user is requested to key in the numbers for the ratio (H\&D)/D, yielding the posterior probability.

\section{Empirical Evaluation}

An experimental predecessor of BasicBayes has been tested empirically (for a detailed description of the study, see Sedlmeier \& Gigerenzer, 1995). Four groups of participants were tested: one received no training and served as a control, two were trained with frequency formats (frequency tree and frequency grid, respectively), and one was given rule training that consisted of teaching participants how to extract the information necessary for Bayes's formula from the problem and to plug it in. Like BasicBayes, the tutor was implemented as a computer program and was kept as similar as possible across training conditions. Participants in the training conditions were tested at four points in time: before training, immediately after training, 1 week after training, and 5 weeks after training. The latter two tests were aimed at examining the transfer and stability of the training effect. All tests were administered on computer.

The participants in all three training conditions gave more correct solutions to Bayesian inference problems than did those in the control group. This effect was, however, much more pronounced in the two frequency conditions than in the rule-training condition. Before training, the median percentage of correct solutions was between $0 \%$ and $5 \%$ in all conditions. Immediately after training, the medians in the frequency conditions were $70 \%$ (frequency grid) and $80 \%$ (frequency tree) as compared with $35 \%$ in the rule-training condition. Five weeks after training, the medians for the two frequency conditions rose to 


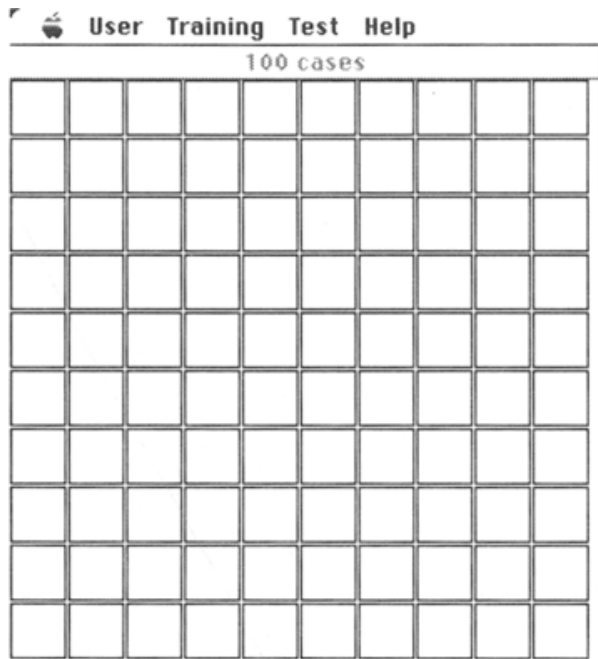

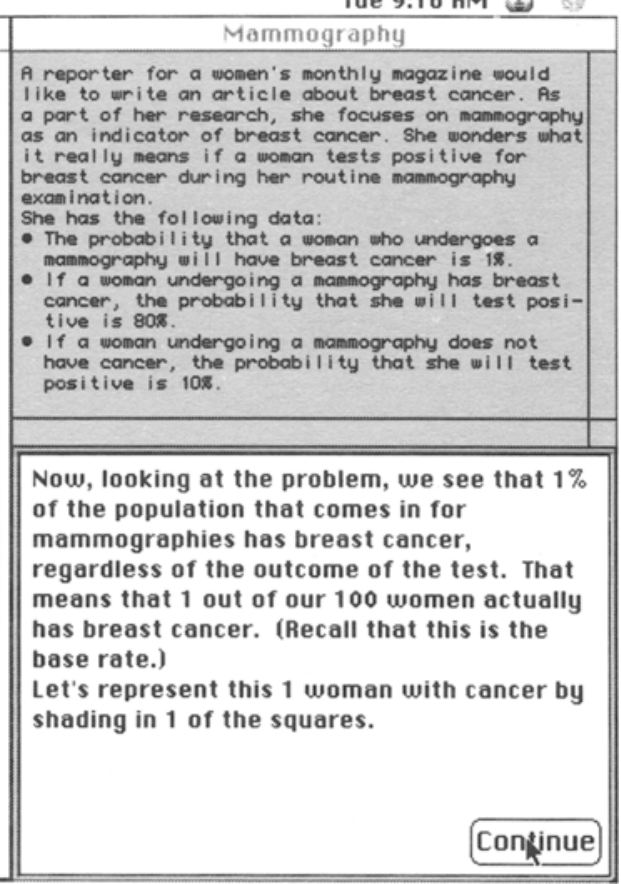

Figure 4. Screen during fixed mode training (mammography problem). The text window displays the text of the mammography problem, the representation window shows a fresh $10 \times 10$ grid, and the tutor window explains the information given in the text in order to prepare the user to work in the representation window.

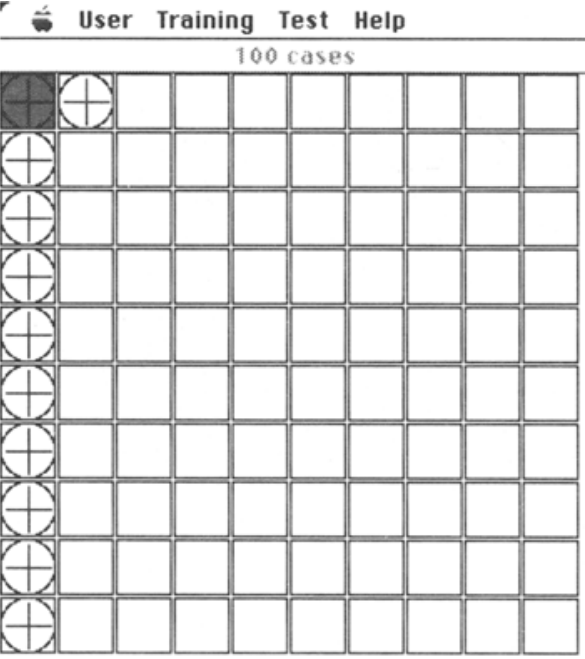

Breast Cancer

Positive Test
Tue 9:17 คM ? Mammography

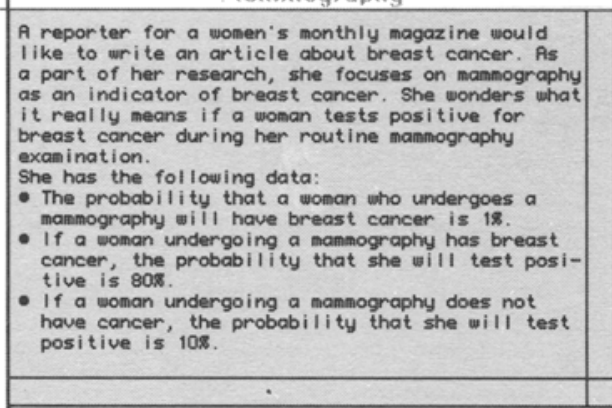

The key to this is the last part of the question, IF SHE TESTS POSITIUE. How many people out of our 100 cases test positive?

One person who actually has cancer tests positive (TRUE POSIIIUE), but so do 10 of the people who do not have cancer (FFLSE POSIIIUES). What is the probability that one of these 11 people who tests positive is the one who has cancer? Quite simply, we know that 1 out of the 11 has cancer. The solution to the problem is: $1 / 11=.09$ ( or $9 \%$ ).

Figure 5. Screen during fixed mode training. BasicBayes has just demonstrated a solution to the mammography problem using a $10 \times 10$ grid. 


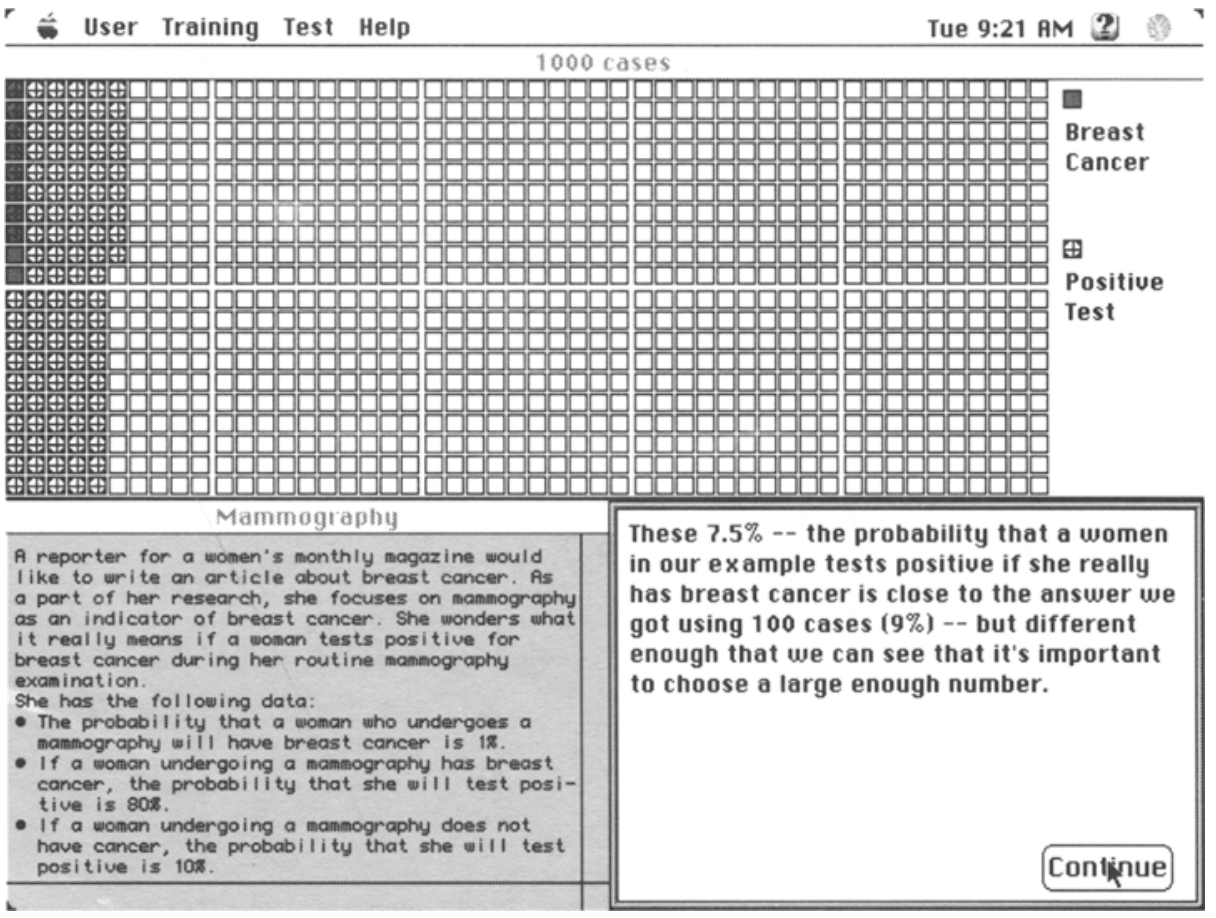

Figure 6. Screen toward the end of fixed mode training. The mammography problem has just been solved a second time, using a $50 \times 20$ grid instead of a $10 \times 10$ grid.

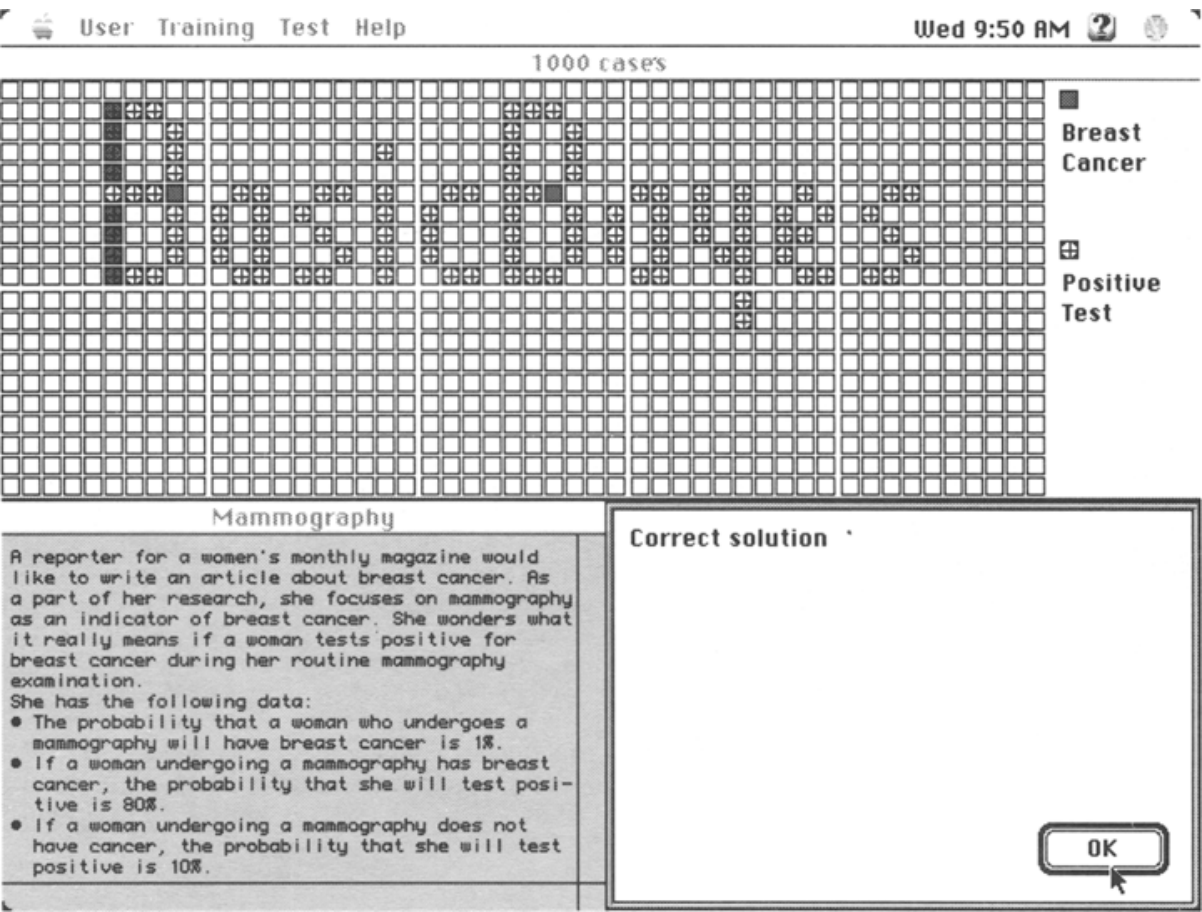

Figure 7. Screen demonstrating that entries in the grid can be made in any order and configuration, as long as the correct numbers of cases are entered. 
$90 \%$, while the corresponding value for the rule training condition was only $20 \%$. Because its effect was neither large nor lasting, rule training was omitted from BasicBayes. According to the theoretical and empirical results of Gigerenzer and Hoffrage (1995), training participants to translate probabilities into frequencies should be sufficient to enable them to solve simple Bayesian tasks. The experimental results reported here are consistent with this view. Having learned once how to perform this probabilityto-frequency translation, the vast majority of participants reliably used it in novel problems long after training had ended. Because BasicBayes is nearly identical to its predecessor in most important respects, one can reasonably expect the current version to be equally effective at teaching Bayesian reasoning.

\section{A Session with BasicBayes}

BasicBayes's predecessor (Sedlmeier \& Gigerenzer, 1995) was embedded in an experimental setup in which participants could not influence the course of training. BasicBayes, in contrast, is fully menu driven and includes several forms of feedback not included in the experimental version. In its current form, BasicBayes can be used for training and testing on 38 Bayesian inference problems. It also allows for the addition of new, custommade problems.

At the beginning of a session, the user is given the opportunity to check whether she is already able to solve the problems in the probability format. A Test menu offers an introduction to using the testing interface as well as direct access to test problems. The user receives immediate feedback as to whether she has solved a problem correctly.

If solving the problems turns out to be difficult for the user, training is in order. The Training menu offers four options-that is, either the fixed or the flexible mode paired with either the frequency grid or the frequency tree. The user can start with either mode but is advised to try the fixed mode first for a thorough introduction to how Bayesian problems are solved in a frequency format. It seems to be a matter of personal preference whether a user chooses the tree or grid version. Use of both the tree and the grid during training might lead to greater flexibility in dealing with probability revision problems after training, although this speculation has not been tested empirically.

During training and test, any number of problems can be chosen by name (problem title) or randomly. It is also possible to switch between training and testing at any point. BasicBayes also provides information that enables the user to evaluate her training progress.

\section{BasicBayes as a Research Tool}

Although the primary aim of BasicBayes is to teach Bayesian reasoning, it can also be used as a tool in research on factors that influence the solution of Bayesian text problems. It allows the researcher to choose a number of problems and have participants work on them without immediate feedback on their solutions. The result is saved in a plain text file and can be inspected by using any text editor.

Although BasicBayes already includes 38 problems, for research purposes it will most likely be necessary to add custom-tailored problems. Accordingly, BasicBayes includes a template that allows the researcher to key in and edit new problems. The template corresponds to the frame-like representation that BasicBayes uses for its problems (see Figure 3). Help instructions are available at every step.

\section{Availability}

BasicBayes is available free of charge for noncommercial use only. The program can be downloaded from the Internet (http://www-psycho.uni-paderborn.de). Transfer of the program via regular mail is also possible. Interested readers should write to the author and enclose a new formatted floppy disk. BasicBayes will run on any 68K Macintosh with System 7 or higher. On a PowerMacintosh, the extensions must be turned off before the program is launched. Although considerable effort has been invested in making BasicBayes error free, there is no guarantee.

\section{CONCLUSION}

BasicBayes is not only based on common sense but on an empirically supported theoretical framework about statistical reasoning (Gigerenzer \& Hoffrage, 1995). The conventional way to teach Bayesian inference is to teach participants how to use Bayes's formula. Instead, BasicBayes teaches people how to translate probability information into frequency information. This kind of training has proved to be quite successful by absolute (in comparison with no training) as well as relative (in comparison with rule training) standards.

BasicBayes illustrates the use of statistical information in everyday life. Although not a formal statistics tutor, it would be a valuable tool in high school and college courses on judgment and decision making. It would also be useful in basic statistics courses. When time to mastery is used as a measure of effectiveness, computerized tutors compare very favorably with other teaching methods (Anderson et al., 1995; Skinner, 1984). Training Bayesian inference in the experimental version of BasicBayes took only 1-2 h (Sedlmeier \& Gigerenzer, 1995). By virtue of its greater flexibility, BasicBayes is expected to achieve the same impressive results as its predecessor in even less time.

\section{REFERENCES}

APPle Computer, INC. (1992). Macintosh Common Lisp reference. Cupertino, CA: Author.

Anderson, J. R., Boyle, C. F., Corbett, A. T., \& Lewis, M. W. (1990). Cognitive modeling and intelligent tutoring. Artificial Intelligence, 42, 7-49.

Anderson, J. R., Corbett, A. T., Koedinger, K., \& Pelletier, R. (1995). Cognitive tutors: Lessons learned. Journal of Learning Sciences, 4, 167-207. 
BAR-HiLLEL, M. (1980). The base rate fallacy in probability judgments. Acta Psychologica, 44, 211-233.

Casscells, W., Schoenberger, A., \& Grayboys, T. (1978). Interpretation by physicians of clinical laboratory results. New England Journal of Medicine, 299, 999-1000.

Christensen-Szalanski, J. J. J., \& Beach, L. R. (1982). Experience and the base-rate fallacy. Organizational Behavior \& Human Performance, 29, 270-278.

Cosmides, L., \& TOOBY, J. (1996). Are humans good intuitive statisticians after all? Rethinking some conclusions from the literature on judgment under uncertainty. Cognition, 58, 1-73.

EDDY, D. M. (1982). Probabilistic reasoning in clinical medicine: Problems and opportunities. In D. Kahneman, P. Slovic, \& A. Tversky (Eds.) Judgment under uncertainty: Heuristics and biases (pp. 249-267). Cambridge: Cambridge University Press.

FiEDLER, K. (1988). The dependence of the conjunction fallacy on subtle linguistic factors. Psychological Research, 50, 123-129.

Fong, G. T., Lurigio, A. J., \& Stalans, L. J. (1990). Improving probation decisions through statistical training. Criminal Justice \& Behavior, 17, 370-388.

GIGERENZER, G. (1994). Why the distinction between single-event probabilities and frequnncies is important for psychology (and vice versa) In G. Wright \& P. Ayton (Eds.), Subjective probability (pp. 129-161). New York: Wiley.

Gigerenzer, G., Hell, W., \& Blank, H. (1988). Presentation and content: The use of base rates as a continuous variable. Journal of Experimental Psychology: Human Perception \& Performance, 14, 513-525.

GigerenZer, G., \& Hofrrage, U. (1995). How to improve Bayesian reasoning without instruction: Frequency formats. Psychological Review, 102, 684-704.

Gigerenzer, G., Hoffrage, U., \& Kleinbölting, H. (1991). Probabilistic mental models. A Brunswikian theory of confidence. Psychological Review, 98, 506-528.

HERTWIG, R. (1995). Why Dr. Gould's homunculus doesn't think like Dr. Gould: The conjunction fallacy reconsidered. Konstanz: HartungGorre.

KEENE, S. E. (1989). Object-oriented programming in COMMON LISP. Reading, MA: Addison-Wesley.
Lindeman, S. T., van den Brink, W. P., \& Hoogstraten, J. (1988). Effect of feedback on base-rate utilization. Perceptual \& Motor Skills, 67, 343-350.

MiNsKY, M. (1975). A framework for representing knowledge. In P. H. Winston (Ed.), The psychology of computer vision (pp. 211-277). New York: McGraw-Hill.

Nathan, M. J., Johl, P., Kintsch, W., \& LeWIS, C. (1989). An unintelligent tutoring system for solving word algebra problems. In D. Bierman, J. Breuker, \& J. Sandberg (Eds.), Artificial intelligence and education (pp. 169-175). Amsterdam: IOS.

NEWMAN, D. (1989). Is a student model necessary? Apprenticeship as a model for ITS. In D. Bierman, J. Breuker, \& J. Sandberg (Eds.) Artificial intelligence and education (pp. 177-184). Amsterdam: IOS.

NisBetT, R. E. (1993). Rules for reasoning. Hillsdale, NJ: Erlbaum

Peterson, C. R., DuCharme, W. M., \& Edwards, W. (1968). Sampling distributions and probability revision. Journal of Experimental Psychology, 76, 236-243.

SCHAEFER, R. E. (1976). The evaluation of individual and aggregated subjective probability distributions. Organizational Behavior \& Human Performance, 17, 199-210.

SedLMEIER, P., \& Gigerenzer, G. (1995). Teaching Bayesian reasoning in less than two hours. Manuscript submitted for publication.

Sedlmeier, P., \& Robles de Acuña-Ponseti, J. (1992). "Intelligente" Hilfe beim Lösen von alltagsnahen Wahrscheinlichkeitsproblemen: Modellierung dynamischer Wissensinhalte für ein flexibles Tutorsystem ["Intelligent" help in solving probability problems with everyday applications: Modeling of dynamic knowledge in a flexible tutor system]. Kognitionswissenschaft, 3, 24-37.

SHAUGHNESSY, J. M. (1992). Research in probability and statistics: Reflections and directions. In D. A. Grouws (Ed.), Handbook of research on mathematics teaching and learning (pp. 465-494). New York: Macmillan.

SKINNER, B. F. (1984). The shame of American education. American Psychologist, 39, 947-954.

(Manuscript received February 28, 1996; revision accepted for publication June $11,1996$. 